\title{
ANALYSIS OF CENTRAL-PLACE SPACE-USE PATTERNS: THE ELASTIC DISC REVISITED ${ }^{1}$
}

\author{
Thomas GetTy \\ Division of Biological Sciences, University of Michigan, \\ Ann Arbor, Michigan 48109 USA
}

\begin{abstract}
Ideas from ecology, economics, and geography are synthesized into a central-place, space-utilization model which is analytically as well as conceptually useful. Incorporation of social and ecological factors extends common home range models, which incorporate only distance. The patch preference and competition coefficients which are "revealed" by regression on actual useintensity patterns indicate the importance of these factors by the degree to which they perturb an appropriate distance-decay function. Proximate factors reveal themselves by deforming a Huxleyan "elastic disc." The analytical value of the model is illustrated by an example from my studies of eastern chipmunks.
\end{abstract}

Key words: central-place foraging; competition; distance-decay function; elastic disc; home range; patchy habitat; space utilization.

\section{INTRODUCTION}

Huxley (1934) introduced the metaphor of an "elastic disc" to describe the flexibility of territorial aggression. This concept implicitly incorporates cost and benefit considerations which have been explicitly modeled by Covich (1976), Orians and Pearson (1979), and others using optimality analyses of space utilization. In contrast to these, most home range models currently available in the mammalian literature are purely descriptive, and they frequently incorporate only locational or distance considerations (e.g., Van Winkle 1975, Dixon and Chapman 1980). This paper develops a general, central-place, space-use model which relates intensity of use of a point in space to three factors: distance from the central place, the microhabitat structure of the area, and the use intensity of that space by conspecific neighbors with overlapping home ranges. The mechanisms incorporated in the model generate flexible space-use patterns which can be described as elastic discs.

The model was developed to serve as a tool for the analysis of the home range patterns of eastern chipmunks (Tamias striatus). This species exhibits a territorial system described as "reversal of dominance with position in space" (Dunford 1970). Established adult individuals have home ranges which are clearly organized around an intensively used, frequently visited central place, which corresponds to the burrow system entrance and/or a favorite perching place (Elliott 1978). Kramer and Nowell (1980) have successfully applied the central-place foraging model of Orians and Pearson (1979) to brief periods of foragingcaching by this species. However, outside of periodic bouts of caching behavior, chipmunk movements and

\footnotetext{
${ }^{1}$ Manuscript received 18 September 1980; revised 6 February 1981; accepted 1 March 1981.
}

space-use intensity show a central-place pattern which cannot be easily interpreted by optimal foraging considerations alone. This lack of fit is probably true of central-place systems in general (c.f. Orians and Pearson 1979). Foraging chipmunks frequently consume items at the site of discovery and often return to the central perch without food and simply sit. The frequent returns to the central perch are probably related to detection of intruding competitors and predators.

This model, and the field study it complements, does not attempt to partition risks and resources, but simply classifies space as being of a certain microhabitat, or patch type. The formulations which follow do not develop an optimal-foraging model. They are designed instead to allow relatively long-term use-intensity patterns to be examined as a function of proximate distance, environmental, and social factors. Many of these ideas have been approached from another perspective in the literature on economic geography. This model is synthesized from ideas in economics and geography, as well as biology, and may serve as a general analytical tool. An example will illustrate its usefulness in my own work with chipmunks. Consideration is restricted to patterns within established home ranges.

\section{Conceptual Development Patchy habitat}

The analysis of patch utilization patterns has developed as an aspect of optimal foraging theory (Pyke et al. 1977). Most studies consider animals which are not attached to a central place, as they move through patches of differing prey abundance (e.g., Smith and Dawkins 1971, Krebs et al. 1974). If such foragers move without prior knowledge (familiarity from memory or sensing at a distance), then patches must be assessed during exploitation, and the problems of 
patch choice and intensity of use resolve into the simpler problem of when to "move on" (Parker and Stuart 1976). However, learning and memory are important processes in foraging behavior (Zack and Falls $1976 a, b, c)$, especially for animals with a stable home range. Familiarity is relevant to risks of predation, as well as to resource acquisition (Metzgar 1967).

Royama (1970) examined diet composition of Great Tits, and considered patches of different prey composition (quality) as well as prey quantity. He developed the general concept of "patch profitability," and concluded that foraging effort should be allocated by profitability, which may in turn be depressed by effort. Proportional matching of relative effort to relative profitability is clearly not optimal if profitabilities are stationary $(100 \%$ of the effort should be directed to the most profitable patch). However, sequential shifts in effort could be expected to follow exploitation and resource depression (Charnov et al. 1976, Zack and Falls 1976a). I use the term "patch type" to mean all discrete patches of a given type, which is equivalent to Royama's use of "niche." He argues that when the profitability of patch type B equals that of type A, "there is no reason for the predator to spend more time in niche $B$ than in niche $A$, since the predator can now take equal profits out of $A$ and $B$. Under these circumstances, the allotment of time between niches $A$ and $B$ would be a matter of chance, and so on the average, and equal amount of time is alloted to each" (Royama 1970: 614). Other things being equal, $A$ and $B$ are substitutable (per unit area), and the predator should show no preference. This relationship can be restated in economic terms: the consumer should be indifferent between $A$ and $B$. Later in this paper, "indifference maps" are constructed by drawing isoclines through areas which are equally attractive.

\section{Competition}

The impact of conspecific competitors, as well as intrinsic productivity, on patch profitability is discussed by Goss-Custard (1977), who combines both aggressive interference and exploitative resource depression into "interference." The present model does not attempt to separate these direct and indirect effects. I will assume, for now, that conspecifics have a negative effect, but for semantic reasons I choose to call this competition rather than interference. This terminology does not exclude the possibility that neighbors affect each other through predators and parasites, as well as resources.

\section{Distance}

Distance forms the foundation of theories on the location of economic activity developed by economists and geographers (Chisholm 1975, Haggett et al. 1977). Although many of these models are conceived in gravitational terms, with centers attracting commerce from surrounding fields, the formulations allow examination of the reverse process. The obvious importance of distance considerations is formally stated in "the principle of least effort" (Zipf 1949) and "the law of minimum effort" (Losch 1954). These predict the occurrence of so-called " distance-decay functions": "the most common of all regularities abstracted from two-dimensional space, . . . they have been identified at all geographical scales from intra-urban shopping habits to international trade transactions, and seem to operate for all forms of spatial interaction" (Chisholm 1975: 112).

\section{Revealed preference}

Rushton (1971) suggests that two classes of variables influence consumer choice: distance and place utility. This is a generalized restatement of Reilly's (1929) "law of retail gravitation": the frequency with which residents of a given area shop in two competing towns is proportional to the towns' respective sizes and is inversely proportional to their relative distances. Place utility is a more general concept than profitability and can, in theory at least, incorporate risks of predation or injury, as Altmann (1974), Covich (1976), and Parker and Stuart (1976) have attempted in their models. Applying the optimality approach (Schoener 1971, Cody 1974, Maynard Smith 1978) to utility functions for purposes of generating predictions would require the quantification of place utility in units of "fitness value" of points in space (McCleery 1978). However, Samuelson (1938) introduced the concept of "revealed preference" to economics in an effort to "purge demand theory of vestigial traces of the utility concept" (Houthakker 1961: 706). Revealed preference theory assumes rational behavior (defined primarily be consistency and transitivity, not consciousness or optimality) and asserts that it is possible to rank objects according to preferences revealed by choices. This idea is not novel to biology. Many approaches to the study of optimal diet involve ranking items (Pyke et al. 1977). Like the "compression hypothesis" (Mac Arthur and Wilson 1967), these assume that preference reflects profitability.

A space utilization pattern is considered a choice, revealing subjective preferences. However, preferences do not necessarily reveal profitability; they simply indicate that the animal behaves "as if" preferred items are more valuable. Modern economics no longer assumes that utility can be measured as an objective property of various options (Mansfield 1975). Preferences reflect subjective utility, which may or may not correspond exactly to actual profitability or "fitness value." In this paper, utility is equated to preference. Circularity only becomes a problem if a strong inferential link is made between preferences and profitability or fitness (c.f. Tversky and Kahneman 1981, contrary to Caraco 1980). It is not necessary to assume preferences are optimal to apply the following model analytically, although "irrational"' behavior would 
certainly limit our ability to analyze behavior. The model can be used analytically by regression on appropriate data. The use of revealed preferences reverses the usual process of assessing the "value" of space a priori and predicting behavior (c.f. Altmann 1974). If animals behave as if certain factors are more important than others, then we can proceed to determine the causes in a variety of ways.

The characteristics of the model are examined and illustrated here by simulations. These simulations simply show the space utilization patterns that are expected if the animal behaves as modeled in the presence of the simulated conditions.

\section{THE ModeL}

To make use of revealed preferences, I propose that at a point $x, y$ within area $\int d A$, "utilization"' $[u(x, y)]$ and "attractiveness" $[a(x, y)]$ have the following relationship:

$$
\frac{a(x, y)}{\int a(x, y) d A}=\frac{u(x, y)}{\int u(x, y) d A} .
$$

Within a homogeneous patch (area $\int d P$ ) that is used in a fine-grained fashion (c.f. Levins 1968):

$$
\frac{a(P) \int d P}{\int a(x, y) d A}=\frac{\int u(x, y) d P}{\int u(x, y) d A},
$$

which can be rearranged to:

$$
a(P)=\frac{1}{\int d P} \int a(x, y) d A\left[\frac{\int u(x, y) d P}{\int u(x, y) d A}\right] .
$$

This equation simply defines the attractiveness of a patch (per unit area) as being proportional to its relative utilization. The relative utilization (the right, bracketed term in Eq. 3) is the "use intensity" of a space, patch, or other item. Other things being equal (i.e., distance and competition, Eqs. 5-7 below), the ratio of the attractiveness of two areas equals the relative preference (Murdoch 1969, c.f. Greenwood and Elton 1979).

From Eq. 1:

$$
a(x, y)=u(x, y)\left[\frac{\int a(x, y) d A}{\int u(x, y) d A}\right] .
$$

In examining revealed preferences, we expect $\int u(x$, $y) d A$ to vary with conditions, and $\int a(x, y) d A$ to be constrained (i.e., conventionally normalized to one). In an analytical situation, use intensity $\left(u(x, y) / \int u(x\right.$, $y) d A$ ) may be used directly, but the concept of attractiveness is developed here to relate use intensity to the revealed preference concept. For simulation purposes, the absolute value of $a(x, y)$ is unimportant; therefore we will ignore differences in total time budget, and observe that $a(x, y)$ is proportional to $u(x$, y) (by definition).

The individuals in the habitat are constrained by some degree of site attachment ("phase 2" of May- nard Smith 1974), returning periodically to a central refuge, perch, caching or feeding site. They experience nonnegligible, additional metabolic costs during locomotion. As stated above, this scenario has the characteristics of a central place forager (e.g., Orians and Pearson 1979), but is not restricted to foraging considerations. Risks may also increase with distance from the center.

To begin, assume that a solitary individual (i) reveals a space utilization pattern in which the use, or attractiveness, of a space is proportional to the preference for that type of space $[p(x, y)]$, weighted by the distance $(d)$ of $x, y$ from the central place:

$$
a_{i}(x, y)=p_{i}(x, y) f_{i}(d) .
$$

This form is analogous to gravity interaction models common in geography (c.f. Haggett et al. 1977) with $p_{i}(x, y)$ analogous to mass, or opportunity at $x, y$ and $a_{i}(x, y)$ analogous to the net "traffic"' $(t)$ to $x, y$ from the center. We expect that in a homogeneous habitat, exploited in a fine-grained fashion, $f_{i}(d)$ will be circularly symmetrical and decrease monotonically with increasing distance. This movement-minimizing condition was suggested by von Thunen (as cited in Haggett et al. 1977), who in 1826 hypothesized concentric rings of land use around an isolated population center. Taylor (1975) categorizes distance-decay functions as variants of his general model: $t=c \cdot \exp (-b \cdot g(d)$, where $g(d)=d^{2}$ in the "normal" model. I will assume for now that $f_{i}(d)$ is a circular normal distribution, such that:

$$
a_{i}(x, y)=p_{i}(x, y) \cdot c_{0} \cdot \exp \left(-c_{0}^{2} d^{2} \pi\right) .
$$

This distribution is a reasonable first approximation for several reasons. It is simple, and the slope is small at the center and at the periphery, where it decreases smoothly rather than ending abruptly. The latter characteristics are found in my chipmunk data, and probably many other home ranges. For simulations, I ignore individual differences in $f_{i}(d)$ and set the dispersion coefficient $c_{0}=0.1$, which is typical for my chipmunks. This value of $c_{0}$ corresponds to $a(d=10$ $\mathrm{m})=0.1 a(d=0)$. The real possibility of individual diferences in preferences is not examined here.

Conspecifics may occupy the habitat. It is hypothesized that these will affect $a_{i}(x, y)$ such that:

$$
a_{i}(x, y)=p_{i}(x, y) \cdot\left[1-c_{1} \sum a_{j}(x, y)\right] \cdot f_{i}(d) .
$$

This multiplicative relationship is similar in intent to the competition coefficients of the Lotka-Volterra equations, but is not directly related to them. It is assumed (though not necessary) that the coefficient of competitive impact $c_{1}$ is positive, as a result of resource depression or interference competition. All else being equal, an increase in the use of a space by one neighbor should result in a decrease by others. However, the relationship between access to space and 

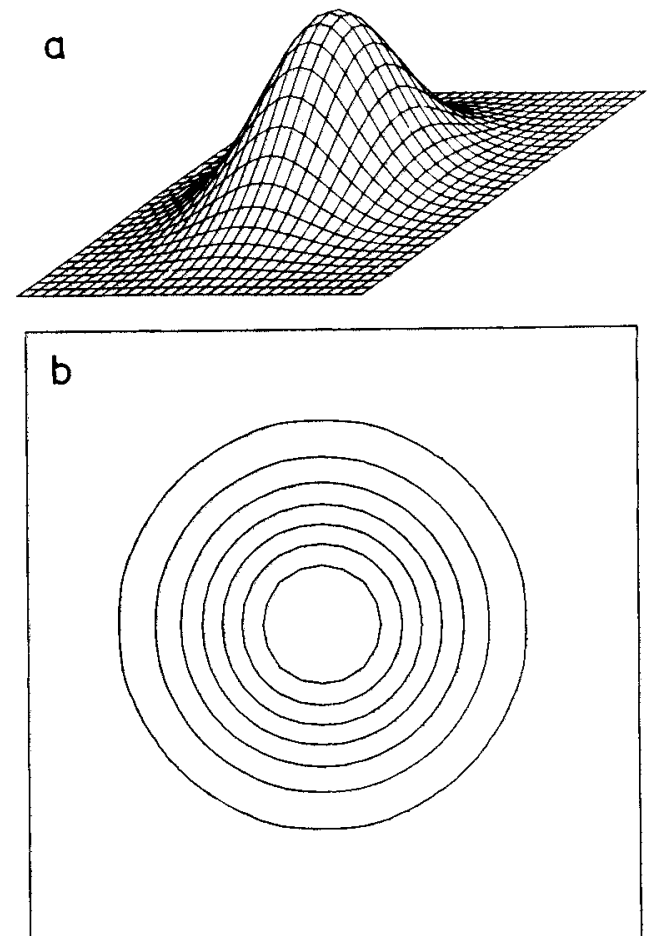

FIG. 1. (a) An orthographic projection of a circularly normal distribution. (b) The contour representation of this distribution beginning at $a=0.1$ and increasing at intervals of 2.0.

access to resources may be complex (c.f. Getty 1981), and therefore a competition coefficient related to space-use intensity may not be a good estimator of "the true competition coefficients" (contrary to Hallett and Pimm 1979). A more realistic consideration of exploitation vs. interference might require that these be distinguished as acting differently. Note that simulated attractiveness may be less than zero, while actual use intensity must be greater than, or equal to, zero.

This specific formulation (Eq. 7) may not be the best in all cases. It is important to hypothesize a distancedecay function which is appropriate in each specific case (species, population, etc.). In this general formulation, the dispersion coefficient $c_{0}$ is in units of metres ${ }^{-1}$, and is inversely proportional to the variance of $f_{i}(d)$. The preference coefficient $p$ is in units of metres, indicating the distance at which attractiveness will drop to a given level. As $p$ increases, attractiveness at a given distance increases, and the distance to a given attractiveness decreases. The other variables and coefficients are dimensionless proportionalities. The partial derivative of the dependent variable on any one independent variable is a function of the remaining two independent variables. The derivative of these partial derivatives against distance shows that $a_{i}$ is increasingly sensitive to changes in $a_{j}$ or $p$ as $d$ approaches zero, and proximity to the central-place in-
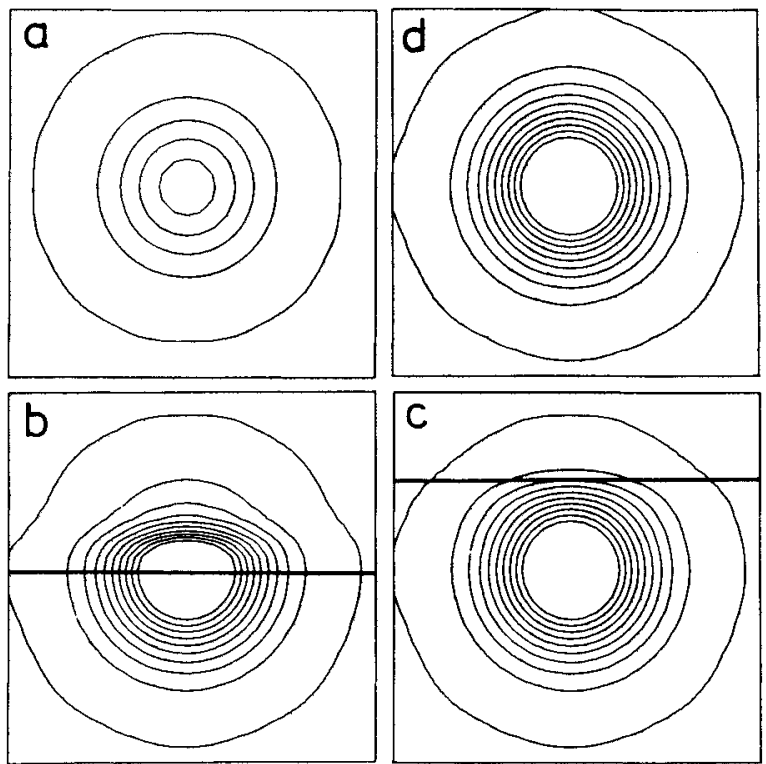

FIG. 2. The impact of a step increase (from 100 to 300) in place utility at differing locations. In (a) the plane is uniformly low and in (d), uniformly high. In (b) and (c), $p=100$ above the line and 300 below the line.

creases. The simulation model transforms a two-dimensional place-utility (preference) map into an abstract plane of expected attractiveness (and hence utilization). Tobler (1963) discusses the usefulness of such transformations in testing locational theory. Isoclines drawn on this plane connect areas of equal attractiveness, and can be interpreted as indifference isoclines. The maps illustrate how $a_{i}$ changes with variations in the other factors. The relationship of changes in $a_{i}$ to adjustments in real time budgets is discussed below. The appropriateness of the "elastic disc" metaphor is obvious when comparing these maps as conditions are varied. If these simulated patterns mimic real patterns, then the modeled mechanisms may resemble actual mechanisms.

\section{Results of Simulations}

For simulation runs, a discrete approximation is used. The $x, y$ plane is a $30 \times 30$ array. Coefficients and the location of individuals are specified. $a_{i}(x, y)$ is computed from Eq. 7 with a simple FORTRAN program. A contouring program (CONTUR, Department of Geography, University of Michigan) is used to draw smooth isoclines on the array of $a$ 's beginning at 0.1 and increasing at intervals of 2.0. The isoclines are indifference contours. Fig. 1 illustrates the relationship between a bell-shaped " $a$ " distribution and the corresponding contour representation. Sequences of changes in parameters show the effect on the simulated attractiveness of points in space. Time budgets are expected to change under these conditions, subject to other constraints. In the long run, changes in the time budget should occur by adding or deleting effort to 

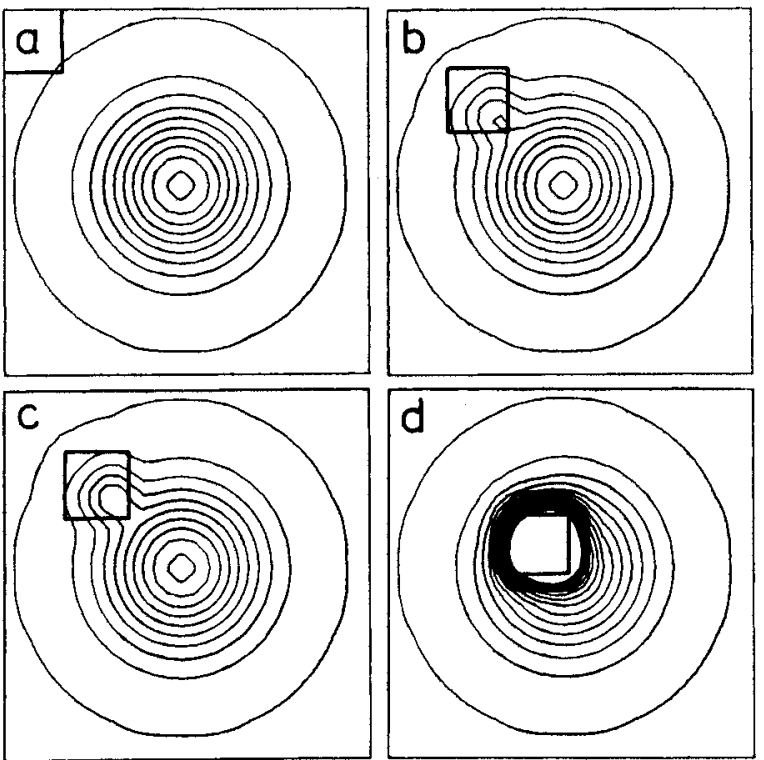

FIG. 3. The impact of a $5 \times 5$ unit patch on the $30 \times 30$ unit field with background $p=200$. The patch has $p=800$ in (a), (b), (d). In (c), p = 1000.

areas in proportion to their simulated attractiveness. Although $a_{i}(x, y)$ should actually be renormalized after each simulation so that $\int a_{i}(x, y) d A$ is equal to one, the relative values of $a_{i}(x, y)$ are informative without forcing us to incorporate this and other constraints related to energy budgets and life histories.

\section{Effect of a patchy habitat}

The sequence illustrated in Fig. 2 simulates the impact of a discontinuity in patch preference, starting with a uniform low value and progressing through a series where a step distribution $[p(x, y)=100$ at $y\rangle$ boundary and $p(x, y)=300$ at $y<$ boundary] moves across the field until a uniformly high distribution occurs. Comparing uniformly low (Fig. 2a) to uniformly high (Fig. 2d), it can be seen that the 0.1 isocline (outermost) and each corresponding contour (at increments of 2.0) is farther from the center in the uniformly "better" habitat. Isoclines which straddle the discontinuity show the tendence for substitution between distance and place utility. These cases approximate a "truncated interaction field" such as Losch (1954) found for the distribution of El Paso bank accounts across the boundary formed by the United StatesMexican border. The trade-off between distance and intrinsic or gross value of items in a choice situation is a key feature of the central-place models of Orians and Pearson (1979) and Schoener (1979). If $p$-values were proportional to energetic profitability and $a$-values proportional to use, then an $a$-value pattern like Fig. $2 \mathrm{~d}$ would represent a larger energy budget than case Fig. 2a. If we allowed the total time budget to vary, a "time minimizer" strategy would result in pro-
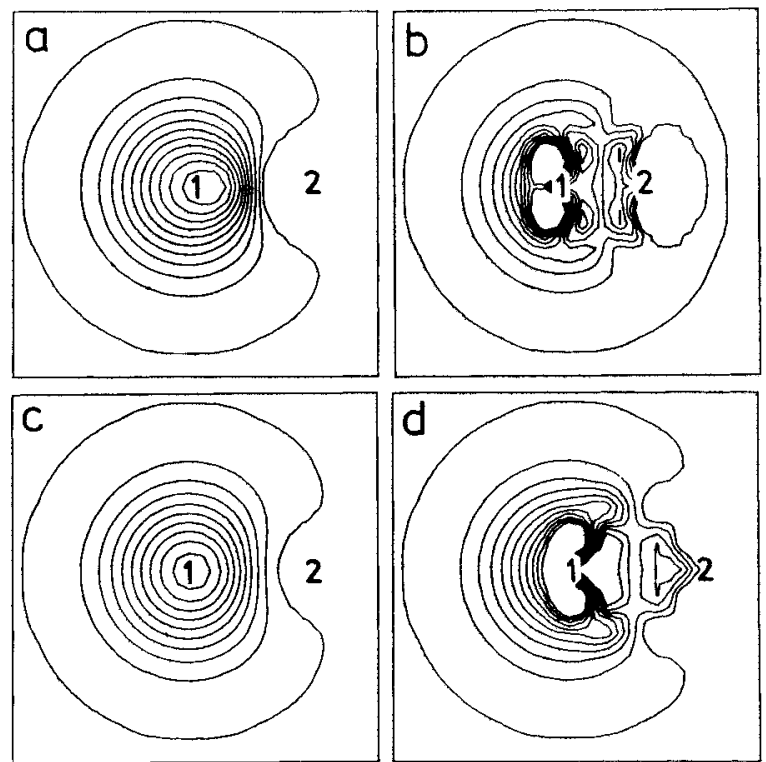

FIG. 4. The impact of a single neighbor in different locations and with varying competitive characteristics. In (a) and (b), the competition coefficient $c_{1}=0.1$, while the separation decreases from 10 to $5 \mathrm{~m}$. In (c) and (d), the separation is $10 \mathrm{~m}$, while $c_{1}$ increases from 0.075 to 0.2 .

gressive contraction of actual use by the retreat of a cumulative percent time boundary (such as $90 \%$ ) along successive contours.

The sequence illusstrated in Fig. 3 models the impact of a $5 \times 5$ unit patch of varying value and at different distances from the central place. Certain combinations of distance and preference result in "patches" of high attractiveness (intensive utilization). Fig. $3 \mathrm{~d}$ indicates the potential lack of correspondence between the central place and the center of activity in a nonuniform habitat (c.f. Smith et al. 1973, Dixon and Chapman 1980).

\section{Effect of conspecifics in a uniform habitat}

Fig. $4 a$ and $b$ simulate the effect of increased proximity between identical individuals (the mirror image map for \#2 is not shown). Fig. $4 \mathrm{c}$ and $\mathrm{d}$ simulate changes in the competition coefficient $c_{1}$. As competitive impact increases slightly $(\mathfrak{c}, a)$, peripheral isoclines pull back from the neighbor. This decreasing overlap results in increased attractiveness near the center (compare the tenth $[a=20.1]$ isocline in a and c).

As separation decreases (a, b), isoclines wrap around behind the neighbor. The small contour which encircles (2) indicates the trade-off between distance and proximity to the neighbor. Locations which are far from (1) and (2) are as attractive to (1) as those closer to (1) but also closer to (2). Both decreased separation $(a, b)$ and large increases in competitive impact $(a, d)$ result in deformation of the central iso- 

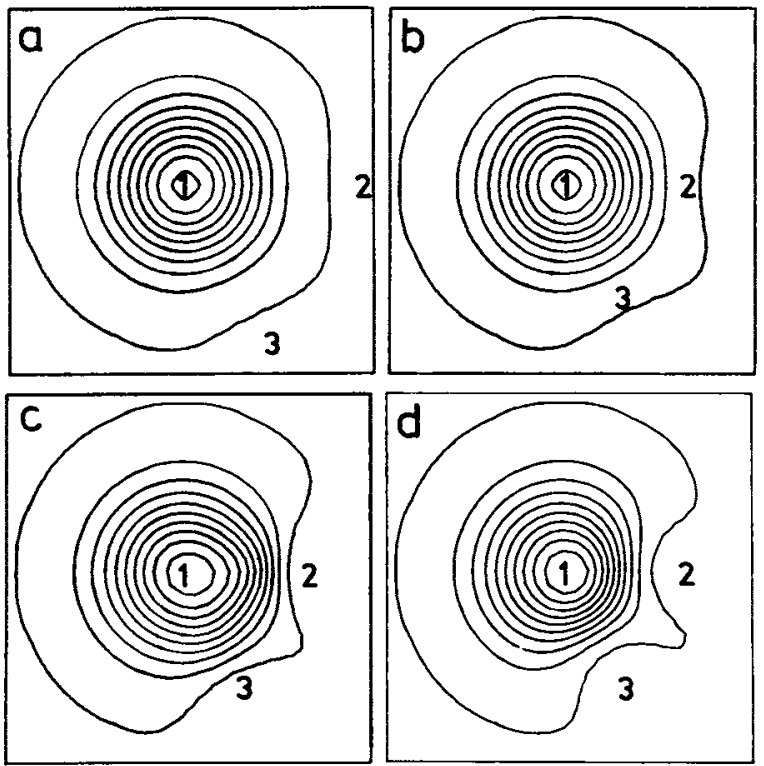

FIG. 5. The impact of two neighbors located to create an equilateral triangle. In (a), $c_{1}=0.1$ and each neighbor is separated by $15 \mathrm{~m}$. In (b), (c), and (d), the separation is $10 \mathrm{~m}$ and $c_{1}$ increases from 0.075 (a) to 0.1 (b) to 0.125 (d).

clines: attractiveness is decreased in proportion to proximity to the neighbor. Note again, however, the increased area within the tenth isocline, as symmetrical escalation of $c_{1}$ results in reduced overlap in $4 \mathrm{~d}$. It is the impact of (1) on (2) which results in the seemingly paradoxical movement of the 0.1 isocline toward (2). As (2)'s central isoclines pull back, the area becomes more attractive to (1). An asymmetrical increase in impact by (2) on (1) would simply result in increased repulsion of (1)'s isoclines and decreased attractiveness throughout (1)'s range. In both $b$ and d, the attractiveness along a transect from (1) to (2) first decreases, then increases slightly before again decreasing. Remember that (2)'s isoclines are the mirror image of (1)'s, and note that the point of inflection is halfway between them. Refer to Eq. 7 and observe that as we move toward (2), $f_{1}(d)$ decreases, and we expect $a_{2}(x, y)$ to increase. However, $a_{2}(x, y)$ is also a function of $a_{1}(x, y)$. The equilibrium solution of Eq. 7 for for $a_{1}$ and $a_{2}$ results in this inflection as a result of the symmetrical trade-off between distance from the resident (1) and proximity to the neighbor (2). This relationship can be examined further, if one wishes, by deriving the solution for $a_{1}$ as a function of $d_{1}, d_{2}$, $c_{0}$, and $c_{1}$, and taking the partial derivative with respect to distance from $(1)\left(d_{1}\right)$.

The sequence illustrated in Fig. 5 simulates the impact of identical animals at the vertices of an equilateral triangle as separation decreases or the competition coefficient $c_{1}$ increases uniformly. No attempt was made to model a resident surrounded by six neighbors, but Fig. 5 suggests that the familiar hexagonal patterns would result from this model. As above, sym-

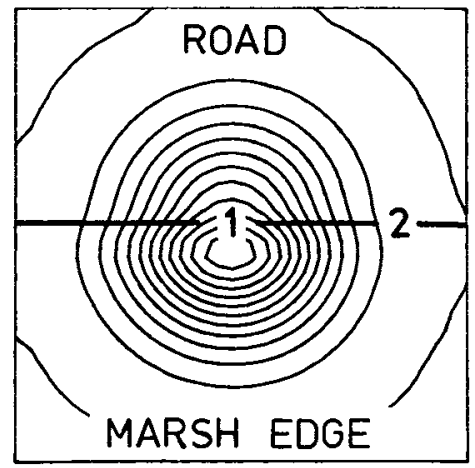

FIG. 6. Simulated composite home range, basec on values derived from seven chipmunks $\left(c_{0}=0.099, c_{1}=-0.026\right.$, $p[$ road $]=164.8, p[$ marsh edge $]=263.4)$. The model accounts for $52 \%$ of the variance in the use-intensity data for this somewhat heterogeneous group $(n=173$ animal $x$ quadrat combinations). The competition coefficient is not significantly different from zero, but distance and patch type have significant effects.

metrical escalation of $c$ 's results in decreased use overlap as neighbors pull back from areas of increased competition (decreased attractiveness). This reduction in overlap results in increased attractiveness of areas near the center.

These few simulations indicate that the model embodies some properties of real space-utilization patterns.

\section{Discussion}

This paper attempts to synthesize ideas from different disciplines into a useful conceptual and analytical tool. The approach rests on the assumption that revealed preferences can be used to determine the importance of hypothesized proximate determinants of behavior. We begin by examining patterns to identify the determinants. Once this is accomplished, we can proceed to ask "why?" by various means.

This model was conceived as a tool to aid in the analysis of data collected during a study of the behavioral ecology of eastern chipmunks (Tamias striatus). It also formalizes conceptions about costs and benefits in a spatial choice situation, so that the implications of these assumptions can be deduced and illustrated. Simulations illustrate the expected trade-offs among distance, patch utility or preference, and competition. Because the model incorporates environmental and social factors, as well as distance considerations, it extends current home range models.

Certain caveats should be mentioned. No attempt has been made to find the "best" formulation in this theoretical presentation. Such a search would be more useful in an analytical situation, where there is a clear criterion for judging various alternatives.

Because real animals move at a finite rate and in a continuous path, we are implicitly considering a longterm, quasi-steady-state pattern. Also as a conse- 
quence of continuous movement paths, a more realistic model might need to deal not only with "intrinsic" characteristics of space, but also with configurations of patches and spatial neighborhoods.

In spite of the simplicity of this model, it has proven useful in my analysis of chipmunk home ranges (Getty 1981). The model was applied to the data by a nonlinear, least-squares regression algorithm. Because patch type is identified as an ordinal affiliation, the dummy variable technique was used to derive the " $p$ " for each patch type. Fig. 6 illustrates the application of the model by simulating a home range, using values derived from the data on seven animals observed in JuneJuly. The idealized, composite individual is centered along the abrupt boundary between a dirt road and the vegetation bordering a marsh. The nearest neighbor in this population is typically $\approx 10 \mathrm{~m}$ away. Deformation of the smooth, symmetrical distance effect illustrates the impact of significant differences in patch preference. Surprisingly, the location and behavior of established neighbors results in the competition factor $\left(c_{1}\right)$ having negligible impact on use intensity. In another paper I demonstrate that spatial time-sharing is facilitated by encounter avoidance between neighbors (Getty 1981). Although the case illustrated in Fig. 6 combines a heterogeneous group of animals, the model accounts for $52 \%$ of the variance in use-intensity. The fit for individuals is usually higher.

\section{ACKNOWLEDGMENTS}

I thank Chris Meacham for help with the computing machine, Neal Oden for his ideas about "attractiveness," and both for their general intellectual stimulation. Many helpful contributions were made by other friends in the Department of Ecology of Evolutionary Biology, including: Diane DeSteven, Pat Dillon, Peter Grant, Hugh McGuiness, Dave Schultz, and the late Donald Tinkle. For their tolerance, as well as help, I thank my doctoral committee: Brian Hazlett; Bobbi Low, Philip Myers, and Beverly Rathcke. Critical editorial assistance was provided by Christine Getty. This work was done in partial fulfillment of the requirements for the degree of Doctor of Philosophy, the University of Michigan, 1980. The work was supported by the Biology Division, the E. S. George Reserve of The Museum of Zoology, and The Rackham School of Graduate Studies.

\section{Literature Cited}

Altmann, S. A. 1974. Baboons, space, time, and energy. American Zoologist 14:221-248.

Caraco, T. 1980 . On foraging time allocation in a stochastic environment. Ecology 61:119-128.

Charnov, E. L., G. H. Orians, and K. Hyatt. 1976. Ecological implications of resource depression. American Naturalist 110:247-259.

Chisholm, M. 1975. Human geography. Penguin, Harmondsworth, Middlesex, England.

Cody, M. L. 1974. Optimization in ecology. Science 183:1156-1164.

Covich, A. P. 1976. Analysing shapes of foraging areas: some ecological and economic theories. Annual Review of Ecology and Systematics 7:235-257.

Dixon, K. R., and J. A. Chapman. 1980. Harmonic mean measure of animal activity areas. Ecology 61:1040-1044.
Dunford, C. 1970. Behavioral aspects of spatial organization in the chipmunk, Tamias striatus. Behaviour 36:215-231.

Elliott, L. 1978. Social behavior and foraging ecology of the eastern chipmunk (Tamias striatus) in the Adirondack Mountains. Smithsonian Contributions to Zoology 256:1107.

Getty, T. 1981. Territorial behavior of eastern chipmunks: encounter avoidance and spatial time-sharing. Ecology 62:915-921.

Goss-Custard, J. D. 1977. Predator responses and prey mortality in redshank, Tringa totanus (L.), and preferred prey, Corophium volutator (Pallas). Journal of Animal Ecology 46:21-35.

Greenwood, J. J. D., and R. A. Elton. 1979. Analysing experiments on frequency-dependent selection by predators. Journal of Animal Ecology 48:721-737.

Haggett, P., A. D. Cliff, and A. Frey. 1977. Locational models. Edward Arnold, London, England.

Hallett, J. G., and S. L. Pimm. 1979. Direct estimation of competition. American Naturalist 113:593-600.

Houthakker, H. S. 1961. The present state of consumption theory. Econometrica 29:704-740.

Huxley, J. S. 1934. A natural experiment on the territorial instinct. British Birds 27:270-277.

Kramer, D. C., and W. Nowell. 1980. Central place foraging in the eastern chipmunk, Tamias striatus. Animal Behaviour 28:772-778.

Krebs, J. R., J. C. Ryan, and E. L. Charnov. 1974. Hunting by expectation or optimal foraging? A study of patch use by chickadees. Animal Behaviour 25:30-38.

Levins, R. 1968. Evolution in changing environments: some theoretical explorations. Princeton University Press, Princeton, New Jersey, USA.

Losch, A. 1954. The economics of location. Yale University Press, New Haven, Connecticut, USA.

Mac Arthur, R. H., and E. O. Wilson. 1967. The theory of island biography. Princeton University Press, Princeton, New Jersey, USA.

Mansfield, E. 1975. Microeconomics: theory and applications. Norton, New York, New York, USA.

Maynard Smith, J. 1974. Models in ecology. Cambridge Press, Cambridge, England.

-1978. Optimization theory in evolution. Annual Review of Ecology and Systematics 9:31-56.

McCleery, R. H. 1978. Optimal behaviour sequences and decision making. Pages $377-410$ in J. R. Krebs and N. B. Davies, editors. Behavioral ecology. Blackwell, London, England.

Metzgar, L. M. 1967. An experimental comparison of screech owl predation on resident and transient white-footed mice (Peromyscus leucopus). Journal of Mammalogy 48:387-391.

Murdoch, W. W. 1969. Switching in general predators: experiments on predator specificity and stability of prey populations. Ecological Monographs 39:335-354.

Orians, G. H., and N. E. Pearson. 1979. On the theory of central place foraging. Pages 155-177 in D. J. Horn, R. D. Mitchell, and G. R. Stairs, editors. Analysis of ecological systems. Ohio State University Press, Columbus, Ohio, USA.

Parker, G. A., and R. A. Stuart. 1976. Animal behavior as a strategy optimizer: evolution of resource assessment strategies and optimal emigration thresholds. American Naturalist 110:1055-1076.

Pyke, G. H., H. R. Pulliam, and E. L. Charnov. 1977. Optimal foraging: a selective review of theory and tests. Quarterly Review of Biology 52:137-154.

Reilly, W. J. 1929. Methods for the study of retail relationships. University of Texas Press, Austin, Texas, USA.

Royama, T. 1970. Factors governing the hunting behaviour 
and food selection by the Great Tit, Parus major. Journal of Animal Ecology 39:619-668.

Rushton, G. 1971. Postulates of central place theory and properties of central place systerns. Geographical Analysis 3:140-156.

Samuelson, P. A. 1938. A note on the pure theory of consumer's behavior. Economica 5:61-71;353-354.

Schoener, T. W. 1971. Theory of feeding strategies. Annual Review of Ecology and Systematics 2:369-404.

1979. Generality of the size-distance relation in models of optimal foraging. American Naturalist 114:902 914.

Smith, J. N. M., and R. Dawkins. 1971. The hunting behaviour of individual great tits in relation to the spatial variation in their food density. Animal Behaviour 19:695706.

Smith, M., B. J. Boize, and J. B. Gentry. 1973. Validity of the center of activity concept. Journal of Mammalogy 54:747-749.

Taylor, P. J. 1975. Distance decay models in spatial inter- action. Concepts and techniques in modern geography, number 2. Geo Abstracts, Norwich, England.

Tobler, W. R. 1963. Geographic area and map projections. Geographical Reveiw 53:59-78.

Tversky, A., and D. Kahneman. 1981. The framing of decisions and the psychology of choice. Science 211:453-458.

Van Winkle, W. 1975. Comparison of several probabilistic home-range models. Journal of Wildlife Management 39: 118-123.

Zach, R., and J. B. Falls. 1976a. Ovenbird (Aves: Parulidae) hunting behavior in a patchy environment: an experimental study. Canadian Journal of Zoology 54:1863-1879.

Zach, R., and J. B. Falls. 1976b. Foraging behavior, learning, and exploration by captive ovenbirds (Aves: Parulidae). Canadian Journal of Zoology 54:1880-1893.

Zach, R., and J. B. Falls. 1976c. Do ovenbirds (Aves: Parulidae) hunt by expectation? Canadian Journal of Zoology 54:1894-1903.

Zipf, G. K. 1949. Human behaviour and the principle of least effort. Cambridge Press, Cambridge, Massachusetts, USA. 